Veritas 日 Scientia

Vol. 9. $\mathrm{N}^{\circ} 1$

Enero - Junio del 2020

ISSN Edición Online: 2617-0639

https://doi.org/10.47796/ves.v9i1.285

\title{
ANÁLISIS DEL SISTEMA DE TRANSPORTE URBANO PARA OPTIMIZAR EL TIEMPO DE VIAJE DEL PASAJERO DE LA CIUDAD DE PUNO-2018
}

\author{
ANALYSIS OF THE URBAN TRANSPORT SYSTEM TO OPTIMIZE THE PASSENGER TRAVEL TIME \\ OF THE CITY OF PUNO-2018
}

Boris Jaime Chique Calderón ${ }^{1}$
Henry Jesús Chique Calderón ${ }^{2}$
Luis Alberto Cabrera Zúñiga $^{3}$

Aceptado: $17 / 05 / 2020$

Publicado online:10/07/2020

\begin{abstract}
RESUMEN
El objetivo de la investigación fue analizar el sistema de transporte urbano de la ciudad de Puno, se propone de infraestructura vial urbano para tener una capacidad vial de acuerdo al tipo de vía y así poder optimizar el tiempo de viaje del pasajero según la tendencia de movilidad, movilidad de origen destino según zonas, movilidad del transporte urbano. Se analizó el recorrido de rutas de transporte público urbano para determinar la velocidad promedio y así poder conocer los tiempos de viajes. La hipótesis que se ha formulado es: con el análisis del sistema de transporte urbano se propone infraestructura vial urbana para optimizar el tiempo de viaje del pasajero de la ciudad de Puno-2018, se analiza la tendencia de movilidad según origen destino, la movilidad del transporte urbano, rutas de transporte urbano público e infraestructura vial urbana. La investigación centró su metodología, bajo un enfoque cuantitativo, diseño no experimental. Se aplicó un cuestionario como un instrumento para el análisis; de los cuales permitieron recoger información y analizar las variables de estudio y efectuar las correlaciones. La muestra estuvo conformada de 384 pasajeros y 8 microbuses que brindan servicio en las principales rutas de transporte público urbano. Como resultado se tiene que el sistema de transporte urbano de la ciudad de Puno es concentrado debido al uso de suelo en la zona céntrica, la movilidad vehicular tiene obstáculos en el flujo debido al uso de la calzada como estacionamiento y que las vías superan su capacidad vial la cual no optimiza los tiempos de viajes en ciudad de Puno para lo cual se requiere de infraestructura vial urbana como son: terminales terrestres, pavimentado de las vías,
\end{abstract}

\footnotetext{
${ }_{1}^{1}$ Maestro en Ingeniería Civil, Consultor de obras, Docente en el Programa de estudios de Ingeniería Civil de la Universidad Nacional del Altiplano. Docente de la Escuela Superior Técnica SENCICO.bchique@unap.edu.pe, bjchique@hotmail.com. ORCID: 0000-0003-0323-7321

2 Maestro en Ingeniería Civil, Consultor de obra, Gerente Regional de Infraestructura del Gobierno Regional de Tacna. ingchique@gmail.com. ORCID: 0000-0001-9945-267X

${ }^{3}$ Doctor en Arquitectura y Urbanismo, académico de la Facultad de Arquitectura y Urbanismo de la Universidad Privada de Tacna, Perú. Lucaz07@hotmail.com. ORCID: 0000-0002-6975-771X
} 
paraderos, declarar zonas rígidas las vías que son utilizadas por las rutas de transporte urbano e implementar un sistema inteligente de gestión de tráfico en las intersecciones principales de la ciudad.

Palabra clave: Análisis, transporte urbano, Pasajero, tiempo de viaje, rutas de transporte urbano, infraestructura vial.

\begin{abstract}
The objetive of the research was to analyze the system urban transport of the city of Puno, it is proposed an urban road infrastructure to have a road capacity according to the type of road and thus be able to optimize the passenger's travel time according to the mobility trend, mobility of destination origin according to areas, mobility of urban transport. The route of urban public transport routes was analyzed to determine the average speed and thus be able to know the travel times. The hypothesis that has been formulated is: with the analysis of the urban transport system, urban road infrastructure is proposed to optimize the passenger travel time of the city of Puno-2018, the mobility trend is analyzed according to destination origin, the mobility of the urban transport, public urban transport routes and urban road infrastructure. The research focused its methodology, under a quantitative approach, nonexperimental design. A questionnaire was applied as an instrument for analysis; of which allowed to collect information and analyze the study variables and make correlations. The sample was made up of 384 passengers and 8 minibuses that provide service on the main urban public transport routes. As a result, the urban transport system of the city of Puno is concentrated due to the use of land in the downtown area, vehicular mobility has obstacles in the flow due to the use of the roadway as parking and that the roads exceed its capacity. road which does not optimize travel times in the city of Puno for which urban road infrastructure is required such as: land terminals, paving of roads, bus stops, declaring rigid areas the roads that are used by urban transport routes and implement an intelligent traffic management system at the main intersections of the city.
\end{abstract}

Keyword: Analysis, urban transport, passenger, travel time, urban transport routes, road infrastructure

\title{
INTRODUCCIÓN
}

El parque automotor del Perú sufrió un incremento indiscriminado, pasó de tener 2 millones 286 mil vehículos, en el 2009, a 5 millones 244 mil, en el 2015. Esta cifra se duplicó, sobre todo, por la entrada al país de una mayor cantidad de motocicletas, mototaxis y camionetas rurales (microbús). Entre los años 2007 a 2017, la zona urbana se ha expandido por el crecimiento poblacional, según el INEI. (censo 2007) la población urbana fue de 120,229 con una tasa de crecimiento de 1.64\%, en el año 2017 la población urbana fue de 129,922 habiendo incrementado en 10 años la cantidad de 9,693 personas, por lo cual aumenta la necesidad de poder movilizarse según un origen y destino de una zona determina a otra para realizar un propósito, lo que demanda un tiempo de viaje que proponiendo infraestructura vial se tenga un óptimo tiempo de viaje en el transporte urbano público. 
En la ciudad de Puno, los problemas más relevantes por el crecimiento de la población, en el parque automotor, expansión del área urbana y aumento de demanda del servicio de transporte de pasajeros: La existencia de una alta congestión vehicular, una demanda de pasajeros en las horas pico y esto a su vez genera malestar, estrés a los usuarios y a los que brindan servicio de transporte urbano y sobre todo demanda más tiempo de viaje de los pasajeros. La falta tanto de actualización, modificación de las rutas de transporte urbano con la finalidad de reestructurar la red vial actual y los paraderos en función al crecimiento poblacional para la asignación de servicio de transporte y descentralizar y descongestionar las zonas críticas del casco urbano de la ciudad mediante el uso de vías alternas.

El servicio del sistema de transporte en la ciudad de Puno, se encuentra centralizado y fraccionado, esta sólo beneficia a una parte de la población porque la mayor oferta se concentra en las rutas que tiene las características de demanda, no existiendo rutas con servicio regular directo, que articulen al centro de la ciudad de Puno con los Barrios o Urbanizaciones principales de la ciudad; los pasajeros realizan más de un viaje diferente para llegar a su destino final y aumenta el gasto que realiza en transporte como también aumenta el tiempo de viaje.

Así mismo, existe una carencia respecto de la asignación de servicios a los centros poblados que han crecido poblacionalmente y se encuentran en proceso de consolidación urbana, como son las zonas rurales y zonas periféricas de la ciudad.

Actualmente el parque automotor que brinda servicio de transporte urbano son: los microbuses (combis), taxis, moto taxis y triciclos, no existe un sistema de transporte masivo, corredores viales ni mucho menos un sistema articulado de transporte; los cuales podrían descongestionar las vías, reducir los tiempos de viajes y brindar un servicio de transporte mucho mejor.

Según la Municipalidad Provincial de Puno existe una alta tasa de transporte informal del orden del $35 \%$, especialmente entre: moto taxis y triciclos que en la práctica no cuentan con zonas de trabajos y paraderos formales en cuanto al servicio que prestan los microbuses (combis) el $15 \%$ es informal generando así una competencia desleal contra los vehículos formales; es importante precisar que más del $80 \%$ de las empresas de transporte que prestan el servicio de transporte urbano no cumplen con el recorrido autorizado y/o no llegan a su paradero inicial y/o final consignado en su tarjeta de circulación.

Actualmente los paraderos iniciales y finales se encuentran ubicados y concentrados dentro del casco urbano de la ciudad de Puno y a la vez son usados como zona de estacionamiento o parqueo de la flota vehicular privado, los cuales generan desorden en el transporte y aglutinamiento de pasajeros provocándose la inseguridad vial y posibles accidentes de tránsito en dichos lugares. Degradando el ornato de la ciudad, generando congestión, desorden y malestar en los vecinos residentes en la zona.

Existe insuficiencia y deficiente señalización horizontal y vertical básico de intersecciones, paraderos y sentidos de tránsito y zonas rígidas en las principales vías de la ciudad. La semaforización en las principales intersecciones de mayor flujo vehicular, no funcionan, o se encuentran en condiciones deplorables, las mismas que se encuentran operando ineficientemente y la falta reparación y mantenimiento.

En la actualidad no se cuenta con un documento de la situación actual del sistemas de transporte urbano de acuerdo a la necesidad de los pasajeros ni a su crecimiento urbano, lo 
que en la presente investigación se buscó por medio de encuestas origen destino de los pasajeros, proponiendo las rutas más óptimas y la implementación de infraestructura vial urbana para su funcionamiento del sistema de transporte, con el análisis del sistema de transporte urbano de la ciudad de Puno se proponer alternativas para optimizar los tiempos de viajes de los pasajeros, beneficiando a los empresarios que brindan el servicio de transporte, los pasajeros y los turistas que visitan la ciudad y así mejorará la calidad de vida de la población de la ciudad de Puno.

Un sistema de transporte urbano debe contar con infraestructura vial tanto para los vehículos como para los pasajeros; las rutas del sistema de transporte más óptimas para los pasajeros, tomando en cuenta el crecimiento de la demanda de pasajeros según su origen y destino que permitan analizar las estrategias en cuanto a la movilidad de los pasajeros, para poder también planificar las inversiones en infraestructura vial y en mejorar el sistema de transporte. De ahí la inquietud por responder a la pregunta ¿Cómo Analizar el sistema de transporte urbano para optimizar el tiempo de viaje del pasajero de la ciudad de Puno?

Tam Wong (2004) propuso un plan de ciclovías para el área metropolitana de Lima y Callao en la que demostró la experiencia viable y necesaria, sobre todo, para los sectores más pobres que ven a la bicicleta como un medio de transporte acorde con su economía, recalcando que para que funcione un sistema de transporte tiene que existir las infraestructuras viales para su óptimo funcionamiento. Camino Bellido y otros (2018) manifiesta que el sector de transporte masivo en buses en Lima Metropolitana es un sector con demanda creciente debido al crecimiento poblacional de la ciudad, pero que como calidad de servicio no ha evolucionado y sigue siendo deficiente, esto hace que el sistema de transporte sea cada vez más caótico impactando en la calidad de vida de las personas, la ciudad de Puno es la capital del departamento y tiene problemas en el transporte urbano, en la actualidad no hay un estudio que pueda solucionar este. Flores Reyna (2017) desarrolló un análisis de la metodología clásica del modelo de planificación del transporte urbano desde el ámbito de la movilidad sostenible, y observó que la no continuidad temporal de los valores de ratio de viaje genera alta variabilidad en los flujos generados o atraídos obtenidos, en la etapa de partición modal, por su parte, presenta limitaciones dada su complejidad tomando en cuenta la diversidad de modos a considerar y que su modelo debe responder a la elección de cada individuo, las limitaciones de los modelos de la etapa de asignación de viajes están también vinculadas a la elección discreta de cada individuo, y se relacionan con el factor subjetivo de la elección de ruta como el desconocimiento de la red, la preferencia por rutas conocidas y la aparición aleatoria de la congestión. Cabe resaltar que la teoría principal de éstos modelos fue preparada para una asignación solo de automóviles, si bien actualmente es posible adaptarla para el transporte público u otros modos, en muestra investigación se construirá y se calibrará un modelo de transporte público propio para la ciudad de Puno. Mendoza Orozco (2013), trabajó con la implementación de la calidad del servicio de transporte urbano colectivo a lo largo de la Pista Juan Pablo II en Managua, al realizar una infraestructura vial se mejora la calidad de servicio y ésta a la vez puede optimizar el tiempo de viaje del pasajero. $Y$ concluye con el acondicionamiento de un nuevo carril exclusivo para el transporte urbano colectivo en la Pista Juan Pablo II; dirigido a la población en general para satisfacer la demanda total del servicio a través de la construcción de infraestructuras adecuadas para tal fin.

Avelar Dueñas (2014), realizó un análisis del proceso de modernización e implicaciones en la movilidad urbana en el sistema de transporte de Tijuana, destacando el estado de desorden 
y de concentración de intereses económicos y políticos que lo han llevado a distar de representar un medio para favorecer la movilidad de las personas y más aun de coadyuvar al sano desarrollo de la ciudad. Marín Cubas (2016), realizó una propuesta urbana del transporte público en la ciudad de Cajamarca, proponiendo el cambio de sentidos viales del transporte público en el centro histórico de Cajamarca, al realizar un estudio de origen destino de los pasajeros se puede cambiar los sentidos de las vías siempre que estas conlleven a optimizar el tiempo de viaje del pasajero.

Los resultados de este trabajo podría ayudar a la municipalidad provincial de Puno a través de la Gerencia de Transporte tomar decisiones en cuanto a la administración del sistema de transporte para poder optimizar los tiempos de viaje, reducir los gastos en pasajes y tener un sistema de transporte ordenado que mejorará el nivel de vida de la población urbana y principalmente de los asentamientos humanos, centros poblados y comunidades que se encuentran ya en la zona urbana, también beneficiará a los turistas tanto nacionales como extranjeros; además fomentará una adecuada y correcta planificación en las inversiones de infraestructura vial urbana y optimización de los recursos en su gasto.

\section{OBJETIVOS}

- Analizar el sistema de transporte urbano para optimizar el tiempo de viaje del pasajero de la ciudad de Puno-2018.

- Determinar el volumen de tránsito para definir el sistema arterial de las vías.

- Proponer infraestructura vial urbana para optimizar el tiempo de viaje del pasajero.

\section{METODOLOGÍA}

El tipo de investigación es aplicada, no experimental, el nivel de investigación es analítico, el cual consiste en describir fenómenos, situaciones, contextos y eventos. El estudio se realizó en la ciudad de Puno (zona urbana), en el año 2018. La población de estudio conformada, por 121,164 personas, con una población de vehículos aproximada:

\begin{tabular}{lll}
\hline Descripción & Tipo de servicio & Cantidad \\
\hline \hline Microbús & Transporte Urbano & 992 \\
\hline \hline Automóvil y station wagon (taxi) & Transporte Urbano & 2,113 \\
\hline \hline Moto taxi & Transporte Urbano & 1,223 \\
\hline \hline Triciclos & Transporte Urbano & 49 \\
\hline Fuente: Municipalidad Provincial de Puno- Gerencia de Transporte &
\end{tabular}

El muestreo fue probabilístico. EL tamaño de la muestra es de 384. Para el estudio se han establecido 21 zonas de las cuales se realiza las encuestas a los pasajeros respecto a su movilidad durante la semana para poder determinar su flujo de los pasajeros de una zona y a otra zona

\section{RESULTADOS}


Los pasajeros realizan su viaje en función de una actividad y en primer lugar está por trabajo un $36.98 \%$, seguido por estudios (30.73\%), en tercer lugar, esta compras y comercio. Las preferencias de viajes son en línea tipo combi con $64.84 \%$, el segundo está el auto propio (vehículo particular) con $13.54 \%$, seguida por moto taxi y triciclos con $10.16 \%$, le siguen a taxi con $8.85 \%$ y finalmente otros medios de transporte con $2.60 \%$

Tabla 1

Tendencia de viajes promedio/día

\begin{tabular}{ccccc}
\hline $\begin{array}{c}\text { Número de } \\
\text { viajes al día }\end{array}$ & Cantidad & $\%$ & Pasajero & Nro. de viajes \\
\hline \hline 0 & 12 & $3.13 \%$ & 3,786 & 0 \\
1 & 8 & $2.08 \%$ & 2,524 & 2,524 \\
2 & 202 & $52.60 \%$ & 63,737 & 127,475 \\
3 & 38 & $9.90 \%$ & 11,991 & 35,971 \\
4 & 73 & $19.01 \%$ & 23,034 & 92,135 \\
5 & 23 & $5.99 \%$ & 7,257 & 36,286 \\
MAS DE 6 & 28 & $7.29 \%$ & 8,835 & 53,009 \\
TOTAL & 384 & $100.00 \%$ & 121,164 & 347,400 \\
\hline
\end{tabular}

Según viajes al día, en primer lugar está con dos viajes (ida y retorno) con 52.60\%, seguida por cuatro viajes con $19.01 \%$, en tercer lugar tres viajes con $9.90 \%$, en cuarto lugar esta más de 6 viajes con 7.29\%, en quinto cinco viajes con 5.99\%, en sexo lugar no viajan con $3.13 \%$ y por ultimo un viaje con $2.08 \%$ donde un $85.68 \%$ no realiza transbordo es decir que de su origen a destino existe la oferta de servicio de transporte, un $9.64 \%$ realiza un transbordo, un $3.13 \%$ realiza dos transbordos, un $1.04 \%$ realiza tres transbordos y un $0.52 \%$ realiza cuatro a mas transbordos. (Fig. 1)

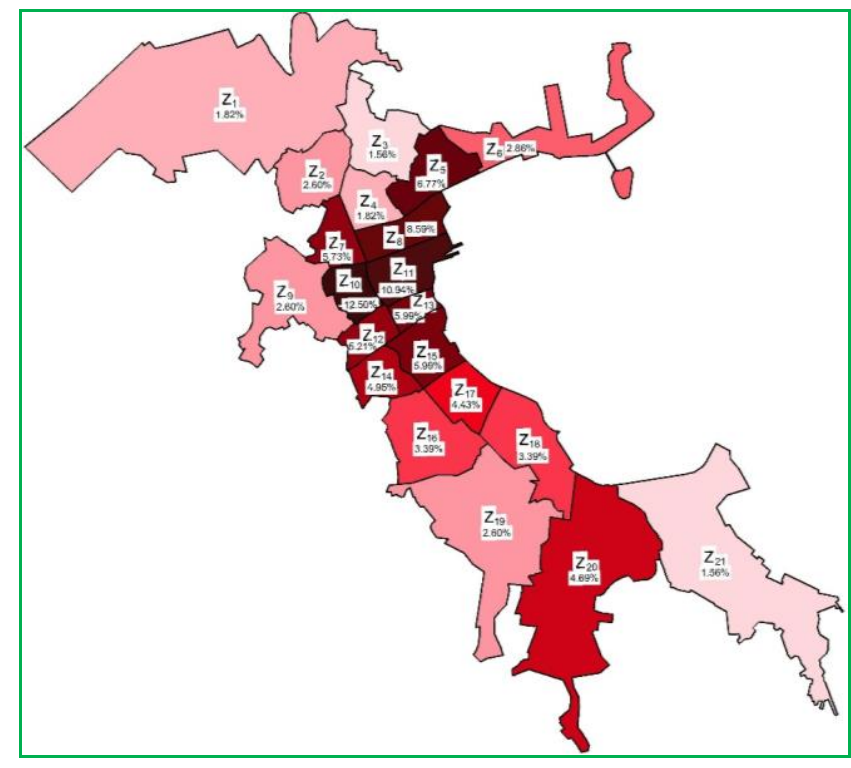

Figura 1. Mapa de destino de la ciudad de Puno

La zona 10 y 11 son las zonas con mayor atracción de pasajeros: en estas zonas se encuentran las instituciones como poder judicial, la municipalidad, gobierno regional, 
instituciones públicas (INEI, OEFA, MTC, ETC) bancos, instituciones educativas, mercado central, restaurantes, centros comerciales, universidades (UPSC, UNAP-FCJP), estadio y el puerto muelle de Puno, entre otros, estos centros hacen que existan fuertes atracción de pasajeros. En 2do lugar está la zona 8 que esta el centro comercial de Plaza Vea, mercado Bellavista, Cajas Municipales, Instituciones Educativas, comercio de ferretería, Galerías Comerciales, edificio administrativo de la UNA, terminal Dorado Express (Puno-Juliaca), terminal San Santiago (Puno-Juliaca), etc.

El criterio para definir los tramos notables de la red vial está en función de la localización espacial e importancia del volumen de transito que circulan por el tramo elegido, de tal manera que sean los tramos de mayor importancia de la red vial.

Las labores de la toma de datos del volumen de transito se llevaron a cabo en días laborables, de lunes a viernes. Se realizó la suma de vehículos por cada hora aforado, con el fin de obtener la hora con mayor demanda vehicular. A continuación, se presenta el cuadro con los volúmenes vehiculares horarios. Análisis de las rutas de transporte urbano público. El contero se ha realizado en las rutas de transporte público urbano principales, se ha realizado un conteo de subida y bajada de pasajeros en los tramos de las rutas de servicio de transporte público.

El Volumen de transito por día en la Av. Juliaca es de 7,918 veh./día, siendo su capacidad máxima de 4000 veh./día según la clasificación en función de su demanda del DG-2018, por lo que el volumen de transito supera su capacidad vial. El Volumen de transito por día en la Av. Sesquicentenario es de 2,253 veh./día, siendo su capacidad máxima de 4000 veh./día. El volumen en la Av. Leoncio Prado -Torre San Carlos es de 4067 veh./día, siendo su capacidad máxima de 4000 veh./día. En la Av. Panamericana Este-Desvió Salcedo es de 4828 veh./día, siendo su capacidad máxima de 4000 veh./día. En la Av. La Torre es de 15,228 veh./día, siendo su capacidad máxima de 6,000 veh./día. En el Jr. Tacna es de 7,516 veh./día, siendo su capacidad máxima de 6,000 veh./día. En el Jr. Deza es de 5,815 veh./día, siendo su capacidad máxima de 6,000 veh./día. En el Jr. Cahuide es de 11,281 veh./día, siendo su capacidad máxima de 6,000 veh./día.

Los microbuses y minibuses de ruta interprovincial son de 1,987 veh./día por lo que se propone un terminal zonal norte y proponer dos carriles adicionales para que la Av. Juliaca sea una autopista de segunda clase para que su volumen de transito no supere su capacidad vial (5,931 veh./día< 6,000 veh./día) y así poder incrementar la velocidad y a la vez optimizar los tiempos de viaje de los pasajeros de la zona norte de la ciudad de Puno. Asimismo un terminal zonal sur en C.P. Jallihuaya para que su volumen de transito no supere su capacidad vial (4,035 veh./día< 4,000 veh./día) y a largo plazo proponer dos carriles adicionales para que la panamericana sur sea una autopista de segunda clase para así poder incrementar la velocidad y a la vez optimizar los tiempos de viaje de los pasajeros de la zona sur de la ciudad de Puno. Declarar zonas rígidas las vías que son utilizadas por las rutas del transporte urbano, para descongestionar e incrementar la velocidad y a la vez optimizar los tiempos de viaje de los pasajeros de la zona céntrica de la ciudad de Puno. Implementar un sistema inteligente de gestión de tráfico en las intersecciones principales de la ciudad de Puno que optimizar los tiempos de viaje de los pasajeros de la de la ciudad de Puno. 
Tabla 2

Prueba de Kolmogorov-Smirnov de origen y destino del pasajero

\begin{tabular}{|c|c|c|c|c|c|c|}
\hline Z & ni & $\mathrm{fi}$ & $\mathrm{Fi}$ & $x i$ & Xi & [Xi-Fi] \\
\hline Z1 & 7 & 0.018 & 0.018 & 18.286 & 0.048 & 0.029 \\
\hline $\mathrm{Z2}$ & 10 & 0.026 & 0.044 & 18.286 & 0.095 & 0.051 \\
\hline Z3 & 6 & 0.016 & 0.060 & 18.286 & 0.143 & 0.083 \\
\hline Z4 & 7 & 0.018 & 0.078 & 18.286 & 0.190 & 0.112 \\
\hline 25 & 26 & 0.068 & 0.146 & 18.286 & 0.238 & 0.092 \\
\hline Z6 & 11 & 0.029 & 0.174 & 18.286 & 0.286 & 0.111 \\
\hline $\mathrm{Z7}$ & 22 & 0.057 & 0.232 & 18.286 & 0.333 & 0.102 \\
\hline Z8 & 33 & 0.086 & 0.318 & 18.286 & 0.381 & 0.063 \\
\hline$Z 9$ & 10 & 0.026 & 0.344 & 18.286 & 0.429 & 0.085 \\
\hline $\mathrm{Z10}$ & 48 & 0.125 & 0.469 & 18.286 & 0.476 & 0.007 \\
\hline $\mathrm{Z11}$ & 42 & 0.109 & 0.578 & 18.286 & 0.524 & 0.054 \\
\hline $\mathrm{Z} 12$ & 20 & 0.052 & 0.630 & 18.286 & 0.571 & 0.059 \\
\hline Z13 & 23 & 0.060 & 0.690 & 18.286 & 0.619 & 0.071 \\
\hline Z14 & 19 & 0.049 & 0.740 & 18.286 & 0.667 & 0.073 \\
\hline $\mathrm{Z15}$ & 23 & 0.060 & 0.799 & 18.286 & 0.714 & 0.085 \\
\hline Z16 & 13 & 0.034 & 0.833 & 18.286 & 0.762 & 0.071 \\
\hline Z17 & 17 & 0.044 & 0.878 & 18.286 & 0.810 & 0.068 \\
\hline Z18 & 13 & 0.034 & 0.911 & 18.286 & 0.857 & 0.054 \\
\hline Z19 & 10 & 0.026 & 0.938 & 18.286 & 0.905 & 0.033 \\
\hline $\mathrm{Z20}$ & 18 & 0.047 & 0.984 & 18.286 & 0.952 & 0.032 \\
\hline Z21 & 6 & 0.016 & 1.000 & 18.286 & 1.000 & 0.000 \\
\hline
\end{tabular}

Tabla 2

Prueba de Kolmogorov-Smirnov del flujo en Av. Juliaca

\begin{tabular}{lcccccc}
\hline $\mathrm{Z}$ & $\mathrm{ni}$ & $\mathrm{fi}$ & $\mathrm{Fi}$ & $\mathrm{xi}$ & $\mathrm{Xi}$ & {$[\mathrm{Xi}-\mathrm{Fi}]$} \\
\hline $7-8$ & 602 & 0.072 & 0.072 & 596.014 & 0.071 & 0.001 \\
$8-9$ & 663 & 0.079 & 0.152 & 596.014 & 0.143 & 0.009 \\
$9-10$ & 598 & 0.072 & 0.223 & 596.014 & 0.214 & 0.009 \\
$10-11$ & 529 & 0.063 & 0.287 & 596.014 & 0.286 & 0.001 \\
$11-12$ & 591 & 0.071 & 0.357 & 596.014 & 0.357 & 0.000 \\
$12-13$ & 575 & 0.069 & 0.426 & 596.014 & 0.429 & 0.002 \\
$13-14$ & 625 & 0.075 & 0.501 & 596.014 & 0.500 & 0.001 \\
$14-15$ & 614 & 0.074 & 0.575 & 596.014 & 0.571 & 0.003 \\
$15-16$ & 609 & 0.073 & 0.648 & 596.014 & 0.643 & 0.005 \\
$16-17$ & 586 & 0.070 & 0.718 & 596.014 & 0.714 & 0.004 \\
$17-18$ & 569 & 0.068 & 0.786 & 596.014 & 0.786 & 0.001 \\
$18-19$ & 609 & 0.073 & 0.859 & 596.014 & 0.857 & 0.002 \\
$19-20$ & 593 & 0.071 & 0.930 & 596.014 & 0.929 & 0.002 \\
$20-21$ & 581 & 0.070 & 1.000 & 596.014 & 1.000 & 0.000 \\
TOTAL & 8344 & & & & & \\
\hline
\end{tabular}


Tabla 3 Prueba de Kolmogorov-Smirnov del flujo en Av. Sesquicentenario

\begin{tabular}{lcccccc}
\hline Hora & \multicolumn{1}{c}{ ni } & $\mathrm{fi}$ & $\mathrm{Fi}$ & $\mathrm{xi}$ & $\mathrm{Xi}$ & {$[\mathrm{Xi}-\mathrm{Fi}]$} \\
\hline $7-8$ & 246 & 0.102 & 0.102 & 172.686 & 0.071 & 0.030 \\
$8-9$ & 213 & 0.088 & 0.190 & 172.686 & 0.143 & 0.047 \\
$9-10$ & 197 & 0.082 & 0.271 & 172.686 & 0.214 & 0.057 \\
$10-11$ & 186 & 0.077 & 0.348 & 172.686 & 0.286 & 0.062 \\
$11-12$ & 173 & 0.071 & 0.420 & 172.686 & 0.357 & 0.062 \\
$12-13$ & 160 & 0.066 & 0.486 & 172.686 & 0.429 & 0.057 \\
$13-14$ & 152 & 0.063 & 0.549 & 172.686 & 0.500 & 0.049 \\
$14-15$ & 172 & 0.071 & 0.620 & 172.686 & 0.571 & 0.048 \\
$15-16$ & 155 & 0.064 & 0.684 & 172.686 & 0.643 & 0.041 \\
$16-17$ & 160 & 0.066 & 0.750 & 172.686 & 0.714 & 0.036 \\
$17-18$ & 155 & 0.064 & 0.814 & 172.686 & 0.786 & 0.028 \\
$18-19$ & 152 & 0.063 & 0.877 & 172.686 & 0.857 & 0.020 \\
$19-20$ & 156 & 0.065 & 0.941 & 172.686 & 0.929 & 0.013 \\
$20-21$ & 142 & 0.059 & 1.000 & 172.686 & 1.000 & 0.000 \\
TOTAL & 2418 & & & & & \\
\hline
\end{tabular}

Tabla 4 Prueba de Kolmogorov-Smirnov del flujo en Av. Leoncio Prado

\begin{tabular}{lcccccc}
\hline Hora & \multicolumn{1}{c}{$\mathrm{ni}$} & $\mathrm{fi}$ & $\mathrm{Fi}$ & $\mathrm{xi}$ & $\mathrm{Xi}$ & {$[\mathrm{Xi}-\mathrm{Fi}]$} \\
\hline $7-8$ & 344 & 0.080 & 0.080 & 308.343 & 0.071 & 0.008 \\
$8-9$ & 352 & 0.082 & 0.161 & 308.343 & 0.143 & 0.018 \\
$9-10$ & 345 & 0.080 & 0.241 & 308.343 & 0.214 & 0.027 \\
$10-11$ & 320 & 0.074 & 0.315 & 308.343 & 0.286 & 0.030 \\
$11-12$ & 323 & 0.075 & 0.390 & 308.343 & 0.357 & 0.033 \\
$12-13$ & 329 & 0.076 & 0.466 & 308.343 & 0.429 & 0.038 \\
$13-14$ & 294 & 0.068 & 0.535 & 308.343 & 0.500 & 0.035 \\
$14-15$ & 269 & 0.062 & 0.597 & 308.343 & 0.571 & 0.026 \\
$15-16$ & 278 & 0.064 & 0.661 & 308.343 & 0.643 & 0.019 \\
$16-17$ & 278 & 0.064 & 0.726 & 308.343 & 0.714 & 0.012 \\
$17-18$ & 310 & 0.072 & 0.798 & 308.343 & 0.786 & 0.012 \\
$18-19$ & 319 & 0.074 & 0.872 & 308.343 & 0.857 & 0.015 \\
$19-20$ & 294 & 0.068 & 0.940 & 308.343 & 0.929 & 0.011 \\
$20-21$ & 260 & 0.060 & 1.000 & 308.343 & 1.000 & 0.000 \\
TOTAL & 4317 & & & & & \\
\hline
\end{tabular}

Tabla 5 Prueba de Kolmogorov-Smirnov del flujo en Av. Panamericana

\begin{tabular}{lcccccc}
\hline Hora & $\mathrm{ni}$ & $\mathrm{fi}$ & $\mathrm{Fi}$ & $\mathrm{xi}$ & $\mathrm{Xi}$ & {$[\mathrm{Xi}-\mathrm{Fi}]$} \\
\hline $7-8$ & 458 & 0.091 & 0.091 & 360.443 & 0.071 & 0.019 \\
$8-9$ & 383 & 0.076 & 0.167 & 360.443 & 0.143 & 0.024 \\
$9-10$ & 367 & 0.073 & 0.240 & 360.443 & 0.214 & 0.025 \\
$10-11$ & 328 & 0.065 & 0.304 & 360.443 & 0.286 & 0.019 \\
$11-12$ & 305 & 0.060 & 0.365 & 360.443 & 0.357 & 0.008 \\
$12-13$ & 326 & 0.065 & 0.429 & 360.443 & 0.429 & 0.001 \\
$13-14$ & 345 & 0.068 & 0.498 & 360.443 & 0.500 & 0.002 \\
$14-15$ & 369 & 0.073 & 0.571 & 360.443 & 0.571 & 0.001 \\
$15-16$ & 376 & 0.075 & 0.645 & 360.443 & 0.643 & 0.002 \\
$16-17$ & 360 & 0.071 & 0.717 & 360.443 & 0.714 & 0.002 \\
$17-18$ & 374 & 0.074 & 0.791 & 360.443 & 0.786 & 0.005 \\
$18-19$ & 368 & 0.073 & 0.864 & 360.443 & 0.857 & 0.006 \\
$19-20$ & 350 & 0.069 & 0.933 & 360.443 & 0.929 & 0.004 \\
$20-21$ & 338 & 0.067 & 1.000 & 360.443 & 1.000 & 0.000 \\
TOTAL & 5046 & & & & & \\
\hline
\end{tabular}




\section{DISCUSIÓN}

El Volumen de tránsito por día en la Av. Juliaca, es de 7,918 veh./día, Av. Leoncio Prado Torre San Carlos es de 4067 veh./día, la Av. Panamericana Este-Desvió Salcedo es de 4912 veh./día siendo su capacidad máxima de 4000 veh./día, supera su capacidad vial; el Volumen de transito por día en la Av. Sesquicentenario es de 2,253 veh./día siendo su capacidad máxima de 4000 veh./día, no supera su capacidad vial. El Volumen de transito por día en la Av. La Torre es de 15,228 veh./día, Jr. Tacna es de 7,516 veh./día, Jr. Cahuide es de 11,281 veh./día siendo su capacidad máxima de 6,000 veh./día, supera su capacidad vial. El Volumen de transito por día en el Jr. Deza es de 5,815 veh./día, siendo su capacidad máxima de 6,000 veh./día, no supera su capacidad vial.

La velocidad promedio del servicio de transporte urbano es de $17.08 \mathrm{~km} / \mathrm{h}$ y el tiempo de viaje está en función de la distancia entre la zona i a zona j. La red vial de las rutas de transporte urbano esta pavimentada con pavimento flexible $(28.09 \mathrm{~km})$, rígido $(40.43 \mathrm{~km}) \mathrm{y}$ sin pavimentar $(5.00 \mathrm{~km}$ ). Se cuenta con $68.52 \mathrm{~km}$ pavimentadas que representa el $93.20 \% \mathrm{y}$ $5.00 \mathrm{~km}$ sin pavimentar que representa $6.80 \%$. Proponemos un terminal zonal en Alto Puno para los vehículos interprovinciales, el volumen de transito se reduciría en la Av. Juliaca a 5931 veh./día y con la construcción de dos carriles adicionales en toda la Av. Juliaca sería una autopista de segunda clase con capacidad máxima de 6,000 veh./día, y así aumentar la velocidad en un promedio de $40 \mathrm{Km} / \mathrm{h}$ y optimizar el tiempo de viaje. Proponemos un terminal zonal en desvío Jallihuaya para los vehículos interprovinciales, el volumen de transito se reduciría en la Av. Panamericana Este a 4076 veh./día y con la construcción de dos carriles adicionales en toda la Av. Panamericana Este sería una autopista de segunda clase con capacidad máxima de 6,000 veh./día, y así aumentar la velocidad en un promedio de $40 \mathrm{Km} / \mathrm{h}$ y optimizar el tiempo de viaje. Proponemos un terminal zonal en la salida Moquegua para los vehículos interprovinciales, el volumen de transito se reduciría en la Av. Leoncio Prado -Torre San Carlos a 3833 veh./día como carretera de primera clase con capacidad de 2000 a 4000 veh./día, y así aumentar la velocidad en un promedio de $40 \mathrm{Km} / \mathrm{h}$ y optimizar el tiempo de viaje. Proponemos zonas rígidas las vías que son utilizadas por las rutas del transporte urbano, para descongestionar e incrementar la velocidad en la línea cortina de la ciudad de Puno. Proponemos un sistema inteligente de gestión de tráfico en las intersecciones principales de la ciudad de Puno que optimizar los tiempos de viaje de los pasajeros de la ciudad de Puno.

Debido que la zona de estudio es la capital del departamento y tiene una tendencia de crecimiento poblacional y expansión urbana se recomienda lo siguiente:

- La Av. Juliaca deber ser una autopista de primera clase que proporcione flujos vehiculares continuos, sin cruces o pasos a nivel y con puentes peatonales.

- La Av. Leoncio Prado y Av. Panamericana Sur deber ser una autopista de segunda clase que proporcione flujos vehiculares continuos, sin cruces o pasos a nivel y con puentes peatonales.

- Que la municipalidad provincial de Puno a través de la Sub Gerencia de Transporte promueva estudios para mejorar el sistema de transporte.

- La señalización horizontal y vertical de las vías deben estar de acuerdo a las normativas vigentes.

- Los vehículos particulares no deben estacionarse en las vías donde circula el servicio de transporte público.

- Realizar estudios que cuente con un sistema de movilidad de los pasajeros. 
- Para estudios futuros realizar el cambio de microbús a vehículos masivos.

- Realizar estudios para implementar transporte con metros, servicios reguladores, servicio expreso y rutas alimentadores.

- Realizar un plan maestro del sistema de transporte para la ciudad de Puno a corto, mediano y largo plazo.

\section{REFERENCIAS BIBLIOGRÁFICAS.}

Agencia de Cooperación Internacional del Japón [JICA]. (2013). Encuesta de recolección de información básica del transporte urbano en el área Metropolitana de Lima y Callao. Informe final (Resumen).: Ministerio de Transportes y Comunicaciones. Lima, Perú

Cabrera, F. (2015). Material virtual del curso "Ingeniería de Tráfico". Pontificia Universidad Católica del Perú. Lima, Perú

Camino Bellido Guillermo Javier y otros (2018) Planeamiento Estratégico Para El Transporte Público Masivo En Buses En Lima Metropolitana (tesis de maestría). Pontificia Universidad Católica del Perú, Lima, Perú.

César Oliver Flores Reyna (2017). Análisis de la metodología clásica del modelo de planificación del transporte urbano desde el ámbito de la movilidad sostenible (tesis de pregrado) Pontificia Universidad Católica del Perú, Lima, Perú.

INEI, Instituto Nacional de Estadística e Informática (2015). Nota de prensa sobre el día mundial de la población. Lima, Perú.

JICA, Agencia de Cooperación Internacional de Japón (2012). Plan maestro urbano para el área de Lima Metropolitana y Callao. Lima: Yachiyo Engineering.

Marvin L. Manheim (1979). Fundamentals of Transportation Systems Analysis, Volume 1 Editorial M.I.T. Press.

Molinero Molinero Ángel R. y Sánchez Arellano (2005), Transporte público, planeación, diseño, operación y administración Toluca, México Universidad Autónoma del Estado de México.

Municipalidad de lima. (2018). instituto metropolitano de Protransporte de Lima. Lima: http://www.protransporte.gob.pe/.

Municipio de León (2009). Plan Maestro de Movilidad del municipio de León hasta 2035. Universidad Iberoamericana, León, México.

Municipio del Distrito Metropolitano de Quito (2018). Sistema Metropolitano de Transporte. Quito: http://www.quito.gob.ec/index.php/municipio/245-sistema-metropolitano-de-transporte.

Ortúzar, J. y Willumsen L. (2008), Modelos de Transporte. Santander, España: Universidad de Cantabria.

Tam Wong, Eduardo, W. (2004). Plan maestro de ciclovías para el área metropolitana de lima y callao (tesis de pregrado). Pontificia Universidad Católica del Perú, Lima, Perú. 